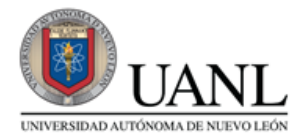

FACPYA
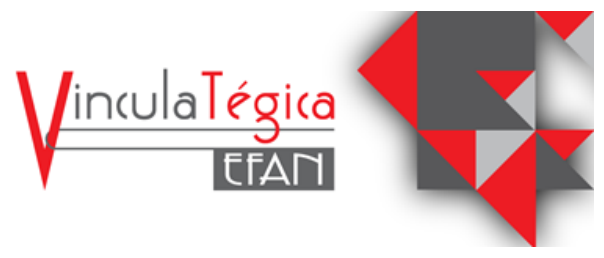

\title{
Perspectiva teórica entre la Inteligencia Emocional y la Resiliencia
}

\author{
María Elvira Cantú González ${ }^{1}$, Amparo Guadalupe Espinosa Domínguez ${ }^{2}$ y Margrory \\ Aymett Mere Ortega ${ }^{3}$ \\ ${ }^{1}$ Universidad Autónoma de Nuevo León, elviracantugonzalez@gmail.com, Avenida universidad s/n Ciudad \\ Universitaria, 8180294532 \\ ${ }^{2}$ Universidad Autónoma de Nuevo León, lupita.espinosa.d@gmail.com, Avenida Universidad s/n Ciudad \\ Universitaria 8115855612 \\ ${ }^{3}$ Universidad San Marín de Porres, mmereo@usmp.pe, Las Calandrias No 151-291 Santa Anita, Lima-Perú \\ 8180294532
}

Información del artículo revisado por pares

Fecha de aceptación: junio-2021

Fecha de publicación en línea: diciembre-2021

DOI: https://doi.org/10.29105/vtga7.1-116

\section{Resumen}

La situación actual derivada del Covid-19 de la pandemia y que afecta a nivel mundial, ha generado cambios de alto impacto en las organizaciones. Este estudio tiene como objetivo analizar los conceptos de inteligencia emocional y la resiliencia, desde los conceptos de diferentes autores. Se examinaron 18 artículos de revistas internacionales y se analizaron desde consideraciones de factores de protección y factores de riesgo encontrándose congruencia entre ambos conceptos. Los resultados confirman dependencia de la inteligencia emocional para llegar a la habilidad de la resiliencia sin embargo el afrontamiento a situaciones adversas solo la resiliencia lo considera al $100 \%$. Y la inteligencia emocional en un $56 \%$. Se concluye que se debe seguir investigando la relación de ambos conceptos.

\section{INTRODUCCIÓN}

Este estudio comparativo tiene como objetivo mostrar la forma en que la resiliencia emana de la práctica de inteligencia emocional; de esta forma contribuye al conocimiento de manera asertiva, dadas las condiciones de pandemia en el mundo, las cuales requieren de adaptación y fortaleza.

\begin{abstract}
The current situation derived from the Covid-19 pandemic, affecting the whole world, has generated high impact changes in organizations. This study has the objective of analyzing the concepts of emotional intelligence and resilience from the perspective of different authors. After reviewing 18 articles from internationally recognized magazines and analyzing from protection factor considerations to risk factors it was found that both concepts to be in similar congruence. The results confirm the dependency of emotional intelligence to be able to develop resilience abilities; however in coping with adverse situations only the resilience considers it $100 \%$ while the emotional intelligence $56 \%$. In conclusion, we must continue investigating the relation between the two concepts.
\end{abstract}

La dinámica social actual a raíz derivada del COVID-19, obliga a las organizaciones a cambiar las formas $y$ estrategias de trabajo, al involucrar y promover, la adaptación en sus colaboradores a nuevas condiciones.

Los factores individuales que justifican una conducta resiliente de algunas 
personas es la inteligencia emocional, por su capacidad de comprender y regular emociones propias y de interacción con los demás (Cassullo y García, 2015; Llorens, Salanova, Torrente, y Acosta, 2013).

\section{MARCO TEÓRICO}

La Organización Internacional del Trabajo (OIT), (2017) frente a la pandemia de COVID19 recomienda una orientación hacia las medidas que deben adoptarse en el ámbito del empleo y el trabajo como prevención en la forma de enfrentar situaciones de crisis y posibilitar la recuperación potencializando la resiliencia.

Siguiendo con la OIT, reconoce el papel fundamental que desempeñan las organizaciones de empleadores y trabajadores en la respuesta a las situaciones de crisis.

Por otro lado, pero en el mismo sentido, la inteligencia emocional es la capacidad que tiene el individuo de reconocer sus propios sentimientos y los ajenos, además de lograr motivarse y manejar las emociones en el mismo y en sus relaciones (Goleman1998). Lo anterior, quiere decir, que mientras una persona sea capaz de manejar inteligentemente sus emociones en escenarios adversos será capaz de adaptarse a ellas, contribuyendo con otros a generar ambientes de cordialidad, que son el preámbulo para el desarrollo de resiliencia.

Dando seguimiento a la idea anterior se encuentra que el término resiliencia se refiere a capacidades, procesos o resultados derivados de una adaptación en un contexto de riesgo o adversidad (Masten 2012, 2014b). La resiliencia se redefine como "el potencial de la capacidad manifiesta de un sistema dinámico para adaptarse con éxito a alteraciones que amenazan la función, supervivencia o desarrollo del sistema" (Masten 2018, pag.187).

\subsection{Definiciones}

Es importante observar cómo, situaciones adversas afectan directa e indirectamente a los individuos. Lo relevante de desarrollar inteligencia emocional es el desdoblamiento que surge hacia la resiliencia. Se vuelve necesario que los colaboradores dentro de la organización estén preparados para adaptarse a nuevas condiciones, para lo cual la inteligencia emocional contribuye favorablemente a enfrentar situaciones adversas, logrando fortalezas resilientes.

\subsubsection{Inteligencia emocional}

En 1920, Edward Thorndie, propone el concepto de inteligencia social como la habilidad para comprender y dirigir a los hombres y actuar sabiamente en las relaciones humanas. Concepto que en. 1998, fue acuñado por Goleman, quien se lleva los créditos como el padre de la inteligencia emocional, al incorporar elementos que parten desde el interior del individuo y desembocan en el ambiente social.

En 1990 Salovey y Mayer, describen la inteligencia emocional como un conjunto de habilidades interrelacionadas sobre las emociones que experimenta el individuo.

El concepto de inteligencia emocional ha sido actualizado de acuerdo a las condiciones que la sociedad demanda. La inteligencia emocional se identifica como un conjunto de habilidades y emociones desde su origen y las competencias que se requieren para manejarlas. Estos conjuntos de competencias llevan al individuo al autoconocimiento, autoregulación, empatía y habilidades sociales, (Según Díaz, 2010). 
Como se observa, cuando surge el concepto de inteligencia emocional, su enfoque se dirigía al reconocimiento y manejo de las emociones propias y de los demás; sin embargo, en la actualidad los teóricos aportan otros elementos que contribuyen al desarrollo de la IE, incorporando el elemento de "competencia".

El concepto de competencia se ha definido como la suma de conocimiento, habilidades y actitudes que son inherentes al ser humano y que lo llevan a desarrollarse de manera efectiva y eficiente en determinados contextos, a través de la experiencia vividas, que le permitan desarrollar y aplicar la inteligencia emocional.

Las competencias de referencia, según Joseph, Jin Newman y Boyle, (2015), son las que promueven en el individuo la regulación y utilización de las emociones en forma positiva beneficiando los contextos en que se desarrolle. Se identifica entonces, que ante la una toma de consciencia o razonamiento a través del cual, el individuo es capaz de tomar conciencia, al racionalizar sus estados emocionales en él y en los demás, logra regular y utilizar las emociones de forma positiva, en beneficio de la organización.

La inteligencia emocional muestra la forma en que los individuos, desarrollan resistencia a estresores, a través de la percepción, comprensión y regulación de sus emociones. Como se mencionó la IE, facilita respuestas adecuadas a la variedad de acontecimientos que el individuo enfrenta diariamente, disminuyendo reacciones o emociones desadaptativas, de manera que posibilita la experiencia de estados de ánimo positivos, reduciendo los negativos. (Cassullo y García, 2015; Llorens, Salanova, Torrente, y
Acosta, 2013). (Pérez, Filella, Alegre, y Bisquerra, 2017).

De las definiciones anteriores se concluye, que a mayor nivel de inteligencia emocional mayores serán las habilidades sociales que el individuo desarrolle. Los vínculos afectivos de calidad llegan a ser un factor de protección frente a la adversidad, lo que se podría considerar como resiliencia, (González, González, y San José, 2017).

\subsubsection{Resiliencia}

El estudio conceptual de la resiliencia va más allá de una teoría, debido a que es parte de la cotidianidad humana y se relaciona con su aspecto comportamental; teniendo en cuenta lo anterior, toda su revisión literaria se enfoca en hechos observados y acontecimientos. (Puentes Quiroga, A. M., Medina Gómez, L. X., \& Castañeda Castaño, M. 2015)

El término resiliencia se describe como una capacidad universal que permite a los seres humanos "prevenir, minimizar o sobreponerse a los efectos dañinos de la adversidad, o anticipar adversidades inevitables". Adicionalmente la resiliencia, fortalece al individuo cuando enfrenta adversidades de la vida, llegando a superarlas e inclusive a dejarse trasformar por la experiencia. Grotberg (1995, 2006).

La resiliencia, se manifiesta frecuentemente entre sujetos que se enfrentan a situaciones de riesgo o adversidad, permitiéndoles conseguir un estado de equilibrio, (Puig \& Rubio, 2011, p. 83).

Por su parte, Sampedro (2009) sostiene que, el término resiliencia es la capacidad de un individuo o de un sistema 
social de vivir bien y desarrollarse positivamente a pesar de las difíciles condiciones de vida $\mathrm{y}$, más aún, de salir fortalecidos.

Los autores anteriormente citados y en coincidenia con, Ruter Zigler, (1987) y Zigler (1991); señalan que la resiliencia es un conjunto de procesos sociales e intrapsíquicos que posibilitan el crecimiento saludable individual, aunque éste haya vivido eventos traumáticos.

En el mismo sentido se encuentran autores que relacionan la resiliencia en términos semejantes como la adaptación positiva mediante un proceso dinámico en contextos adversos y salir fortalecidos ante la adversidad, (Luthar 2001); (Cyrulnik 2005); (Vanistendael 2003).

En los contextos de riesgos o adversidades, que en determinado momento promueven nuevas capacidades y producen resultados satisfactorios se denomina resiliencia. Unos años mas tarde, el termino resiliencia es redefinido como "el potencial de la capacidad manifiesta de un sistema dinámico para adaptarse con éxito a alteraciones que amenazan la función, supervivencia o desarrollo del sistema" (p. 187), Masten (2012, 2014b);( 2018).

Por otro lado, pero en el mismo sentido la Asociación Americana de Psicología define la resiliencia como el proceso logra que el individuo se recupere y fortalezca, de experiencias difíciles, logrando incluso, la competencia de adaptación a circunstancias adversas, traumas o tragedias como fuentes generadoras de estrés (2018).

De acuerdo con las definiciones antes descritas, se concluye que la resiliencia es una competencia que el individuo deberá desarrollar, al percibir los resultados constructivos favorables que puede obtener.

Es importante señalar, que el individuo en ocasiones, no reconoce sus capacidades hasta que se encuentra ante sucesos estresantes o traumáticos que lo llevan al límite de sus capacidades, lo que promueve la acción para resolver.

\subsection{Factores de riesgo protección.}

La resiliencia se considera como una relación entre el individuo, sus experiencias pasadas, que tienen que ver con los aspectos culturales, políticos, económicos y sociales, (Manciaux, citado por Bacca et al. 2014). Donde estas relaciones se determinan por los factores de riesgo y protección. Los factores riesgo fungen como elementos desestabilizadores tales como: maltrato, enfermedades, adicciones y eventos traumáticos e inesperados, que generan incertidumbre como el COVID-19.

Entre los factores de protección se encuentran: la sana autoestima, la manera en que el individuo socializa, el sentido del humor, visión de un proyecto claro de vida, autoestima, inteligencia emocional, autocuidado, optimismo, espiritualidad, esperanza, actitud positiva y redes de apoyo. (Manciaux, citado por Bacca, 2014).

Según Leandro (2017), los factores de protección son: "aquellas circunstancias, características, condiciones y atributos vinculados al comportamiento prosocial, que potencian las capacidades de un individuo para afrontar con éxito determinadas situaciones adversas" (p.2).

Por último, los individuos en su diversidad reaccionan a los factores de riesgo dependiendo de los factores de protección que 
durante su vida hayan desarrollado.

\section{MÉTODO}

Revisión teórica y análisis de 18 artículos publicados en revistas digitales, y plataformas como Dialnet, Google Academic y universidades de Estados Unidos y Europa.

Se diseñó tabla de definiciones de diferentes autores, donde se analizaron elementos como los factores de protección: Capacidad, habilidad, y Factores de riesgo en situaciones adversas, donde se determinaros algunas

\section{RESULTADOS}

Este estudio muestra la forma en que la práctica de la inteligencia emocional, en situaciones adversas o retadoras, contribuye al desarrollo de comportamientos resilientes.

Un hallazgo interesante es que, la resiliencia y la inteligencia emocional muestran características diferentes en todos los seres humanos, por lo tanto, no todos las desarrollan. Aunado a lo anterior, se encuentra que los factores de protección llevan al individuo a responder de determinada manera, dependiendo de los factores de riesgo que el similitudes.

Los resultados se midieron en porcentajes que permitieron mostrar la información con su respectivo análisis.

Se diseñó un modelo comportamental en el cual se muestra como los individuos transitan por diferentes escenarios adversos contribuyen a su fortalecimiento. Ver fiura 1.

\section{Figural. Modelo comportamental de inteligencia emocional y resiliencia.}

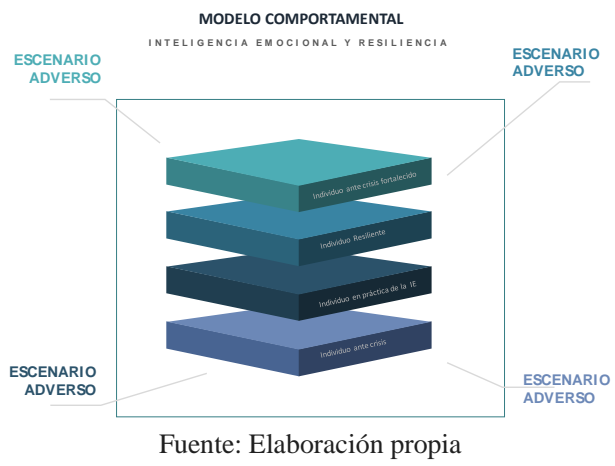

propio individuo identifica.

El estudio muestra que el $33 \%$ de las definiciones consideran la IE como una habilidad, un 55\% como competencia y un $11 \%$ concepto general.

Relacionado con las definiciones de resiliencia un $11 \%$ la definen como habilidad y un $78 \%$ como competencia quedando un $11 \%$ como un proceso.

En cuanto a si se consideran las situaciones de riesgo en las definiciones se encontró que en IE solo el 56\% lo considera; y en las de resiliencia el $100 \%$. 


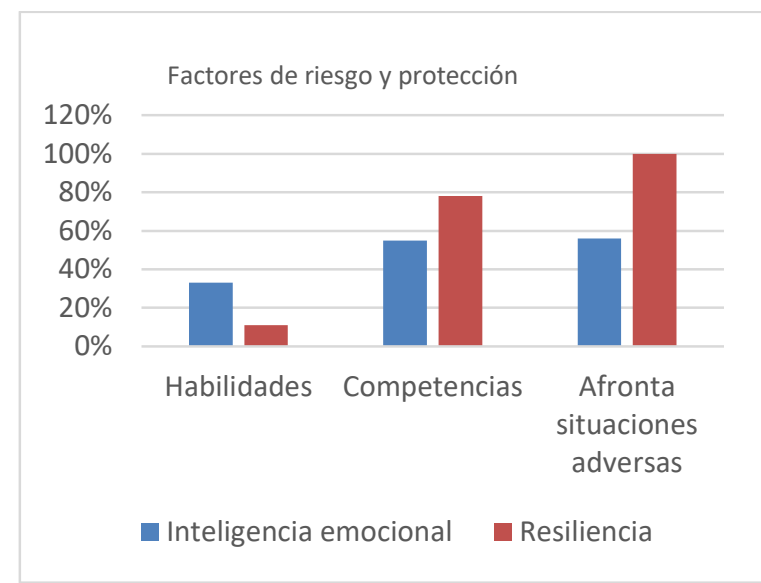

Los resultados del análisis de resiliencia tienen relación semejante al de inteligencia emocional e incluso cierta dependencia.

Sin embargo, la inteligencia emocional no necesariamente se desarrolla en situaciones adversas y la resiliencia siempre.

\section{CONCLUSIONES}

Este estudio muestra la relación o dependencia de la inteligencia emocional que lleva a la resiliencia, sin embargo, diferentes autores no cuentan con una definición homologada de la forma en que depende una de la otra, independientemente de la correlación entre ambas.

El estudio permite fundamentar afinidades entre los dos conceptos: factores de protección (capacidades, habilidades), y factores de riesgo (adaptación en diferentes contextos adversos) y las consideraciones de afrontarlos para salir fortalecidos.

Mi punto de vista es que existe dependencia de la inteligencia emocional y la resiliencia y coincido con Belykh, A. (2018) donde resume que el estudio de la resiliencia ha permitido descubrir las características del individuo, su contexto y procesos psico-sociales que facilitan un manejo exitoso de situaciones adversas o estresantes.

Por otra parte existe relación con la inteligencia emocional la cual estudia a las personas exitosas desde sus rasgos de carácter o capacidades de racionalización emocional y figura como un complemento metodológico basado en los procesos cognitivos que estructura una posible acción para potenciar, entre otras, las cualidades recomendadas por los investigadores de la resiliencia, (Belykh, A. 2018)

La vinculación de estas perspectivas ofrece un marco de referencia para el diagnóstico e intervención en el desarrollo del individuo.

En conclusión, este estudio muestra un camino hacia una nueva línea de investigación, que contribuya a la identificación de factores de riesgo y protección que un individuo deberá identificar y que contribuya a la práctica de la IE y por lo tanto se desencadenen los comportamientos resilientes, necesarios por dinámica actual y retadora que las circunstancias demandan. 
Tabla: 1 comparativa de inteligencia emocional y resiliencia Inteligencia emocional

\begin{tabular}{|c|c|c|c|c|}
\hline \multicolumn{5}{|c|}{ Inteligencia emocional } \\
\hline Año & Autor & Definición & $\begin{array}{l}\text { Factores de protección: } \\
\mathrm{C}=\text { Capacidad } \\
\mathrm{H}=\text { Habilidad }\end{array}$ & $\begin{array}{l}\text { Factores de } \\
\text { Riesgo/Situació } \\
\mathrm{n} \text { adversa }\end{array}$ \\
\hline $920^{1}$ & Edward Thorndike & $\begin{array}{l}\text { La Inteligencia Social y la define como la } \\
\text { habilidad para comprender y dirigir a los hombres } \\
\text { y actuar sabiamente en las relaciones humanas, lo } \\
\text { que ahora podemos considerar como un } \\
\text { antecedente de lo que hoy es conocido como } \\
\text { Inteligencia Emocional }\end{array}$ & $\mathrm{H}$ & \\
\hline 1990 & Salovey y Mayer & $\begin{array}{l}\text { Aportan a la definición de inteligencia emocional } \\
\text { describiéndola como un conjunto de habilidades } \\
\text { interrelacionadas sobre las emociones } \\
\text { experimentadas por los individuos. }\end{array}$ & $\mathrm{H}$ & \\
\hline 1997 & BarOn & $\begin{array}{l}\text { La inteligencia emocional es un conjunto de } \\
\text { habilidades personales, emocionales y sociales y } \\
\text { de destrezas que influyen en nuestra habilidad para } \\
\text { adaptarse y enfrentar a las demandas, y presiones } \\
\text { del medio. }\end{array}$ & $\mathrm{H}$ & $\mathrm{X}$ \\
\hline 1998 & Goleman, & $\begin{array}{l}\text { Es la capacidad de reconocer nuestros propios } \\
\text { sentimientos y los ajenos, de motivarnos y de } \\
\text { manejar bien las emociones, en nosotros mismos y } \\
\text { en nuestras relaciones. }\end{array}$ & $\mathrm{C}$ & \\
\hline 1998 & Cooper & $\begin{array}{l}\text { Es la fuente primaria de la energía humana, la } \\
\text { autenticidad, aspiración y empuje que activan los } \\
\text { más íntimos valores y propósitos en la vida y los } \\
\text { transforman en cosas en que se piensa, en cosas } \\
\text { que se viven. }\end{array}$ & $\mathrm{C}$ & $X$ \\
\hline 2008 & Mayer et al., & $\begin{array}{l}\text { Es la capacidad de participar en un procesamiento } \\
\text { sofisticado de información sobre las emociones } \\
\text { propias y de los demás y la capacidad de utilizar } \\
\text { esta información como guía para el pensamiento y } \\
\text { el comportamiento. }\end{array}$ & $\mathrm{C}$ & \\
\hline 2009 & (Fernández y Extremera & $\begin{array}{l}\text { La Inteligencia emocional nos permite disminuir } \\
\text { la intensidad y la frecuencia de los estados de } \\
\text { ánimo negativos provocados por los } \\
\text { acontecimientos adversos de vida cotidiana. }\end{array}$ & & $X$ \\
\hline 2015 & $\begin{array}{l}\text { Cassullo y García, } \\
\text { Llorens, Salanova, } \\
\text { Torrente, y Acosta, }\end{array}$ & $\begin{array}{l}\text { La inteligencia emocional explica como algunos } \\
\text { individuos se vuelven más resistentes a los } \\
\text { estresores a través de su capacidad de percibir, } \\
\text { comprender y regular sus emociones. }\end{array}$ & $\mathrm{C}$ & $\mathrm{X}$ \\
\hline 2017 & $\begin{array}{c}\text { (Pérez, Filella, Alegre, } \\
\text { y Bisquerra }\end{array}$ & $\begin{array}{l}\text { La inteligencia emocional está relacionada con los } \\
\text { procesos de adaptación, facilitando respuestas } \\
\text { adecuadas a los diferentes acontecimientos que } \\
\text { una persona ha de afrontar en su vida diaria, } \\
\text { disminuyendo las reacciones emocionales } \\
\text { desadaptativas, posibilitando la experimentación } \\
\text { de estados de ánimo positivos y reduciendo la } \\
\text { incidencia de los negativos. }\end{array}$ & $\mathrm{C}$ & $X$ \\
\hline & & Resiliencia & & \\
\hline 1995 & Grotberg & $\begin{array}{l}\text { Señala el concepto de la resiliencia como una } \\
\text { capacidad universal que permite a los seres } \\
\text { humanos "prevenir, minimizar o sobreponerse a } \\
\text { los efectos dañinos de la adversidad, o anticipar } \\
\text { adversidades inevitables" }\end{array}$ & $\mathrm{C}$ & $X$ \\
\hline 2003 & Vanistendael & $\begin{array}{l}\text { Define la resiliencia como una capacidad universal } \\
\text { que permite a una persona, grupo o comunidad } \\
\text { impedir, disminuir o superar los efectos nocivos de } \\
\text { la adversidad." (2003, p. } 18)\end{array}$ & $\mathrm{C}$ & $\mathrm{X}$ \\
\hline
\end{tabular}


Relacionan la resiliencia en términos semejantes como la adaptación positiva mediante un proceso dinámico en contextos adversos; así como también la capacidad de los seres humanos que han sido sometidos a situaciones difíciles superar e incluso salir fortalecidos ante la adversidad.

Define el término resiliencia como la capacidad de un individuo o de un sistema social de vivir bien y

2009 Sampedro

2011 Hjemdal y col.

2013 Santos

2012 ,

$2014 b$

Masten

Asociación Americana

Bonnano

de Psicología desarrollarse positivamente a pesar de las difíciles condiciones de vida $\mathrm{y}$, más aún, de salir fortalecidos y ser transformados por ellas.

Informan que la resiliencia individual contribuye de forma significativa y negativa a la predicción de depresión, ansiedad, estrés y síntomas obsesivocompulsivo.

Define la resiliencia como una actitud vital positiva a pesar de las circunstancias difíciles por las que atraviesa una persona, y representa el lado positivo de la salud mental. Consiste también en saber aprender de la derrota y transformarla en oportunidad de desarrollo personal

Masten (2018) redefine la resiliencia como "el potencial de la capacidad manifiesta de un sistema dinámico para adaptarse con éxito a alteraciones que amenazan la función, supervivencia o desarrollo del sistema" (p. 187).

Defina la resiliencia como un proceso de recuperación de experiencias difíciles y adaptarse bien ante la adversidad, el trauma, la tragedia, las amenazas o fuentes importantes de estrés.

La resiliencia se define como una trayectoria estable de funciones saludables después de un evento altamente adverso, esto lo deduce de su primera definición del 2002 en donde señala que la resiliencia incluye dos aspectos relevantes: resistir el suceso y rehacerse del mismo" (Bonano, Wortman et al, 2002 p.1150). $\begin{array}{ll}\text { C } & \text { X } \\ & \\ \text { C } & \text { X } \\ & \\ \text { H } & \text { X }\end{array}$

C

X

C

X

$\mathrm{X}$

$\mathrm{C}$

X
$\mathrm{X}$

$\mathrm{X}$

$x^{2}$

\section{REFERENCIAS}


Acosta, H., Torrente Barberá, P., Llorens, S., \& Salanova, M. (2013). Prácticas organizacionales saludables: Un análisis exploratorio de su impacto relativo sobre el engagement con el trabajo.

Bar-On, R. (1997a). El Inventario de Cocientes Emocionales ( EQ-i ): una prueba de inteligencia emocional . Toronto, Canadá: Multi-Health Systems, Inc.

Belykh, A. (2018). Resiliencia e inteligencia emocional: conceptos complementarios para empoderar al estudiante. Revista Latinoamericana de Estudios Educativos, 48(1), 255-282.

Cassullo, G.L., y García, L. (2015). Estudio de las Competencias Socio Emocionales y su Relación con el Afrontamiento en Futuros Profesores de Nivel Medio. Revista Electrónica Interuniversitaria de Formación del Profesorado, 18(1), 213-228. doi:10.6018/reifop.18.1.193041

Cooper, R. (1998). La inteligencia emocional aplicada al liderazgo. Colombia. Norma.

Díaz, D. y. (2010). Inteligencia emocional aplicada en la venta. España: Kolima.

DiGrazia, J. C., Ziegler, J., \& Mueller, R. (1987, December). An Ada distributed multiprocessor executive: from conceptualization to implementation. In Proceedings of the 1987 annual ACM SIGAda international conference on Ada (pp. 147-156).

Goleman, D. (1998). Working with emotional intelligence. Bantam.

Grotberg, E. H. (2003). What is resilience? How do you promote it? How do you use it? In E. H. Grotberg (Ed.), Resilience for today: Gaining strength from adversity (pp. 1-30). Westport, CT: Praeger Publishers.

Joseph, D. L., Jin, J., Newman, D. A., \& O'Boyle, E. H. (2015). Why does self-reported emotional intelligence predict job performance? A meta-analytic investigation of mixed EI. Journal of Applied Psychology, 100(2), 298.

Luthar, S. S. (1998, August). Resilience among at-risk youth: Ephemeral, elusive, or robust? Boyd McCandless Young Scientist Award presentation, 106th Annual Convention of the American Psychological Association, San Francisco.

Masten A.S. Resilience theory and research on children and families: past, present, and promise. Journal of Family Theory \& Review. 2018;10(1):12-31.

Morgan, J. (2016). La resiliencia y su relación con el desempeño laboral. Revista Fidélitas, 6, 12-12.

Pérez Escoda, Filella, Alegre y Bisquerra (2012). Desarrollo de la competencia emocional de maestros y alumnos en contextos escolares. Electronic Journal of Research in Educational Psychology, 10(3), pp: 1183-1208

Puentes Quiroga, A. M., Medina Gómez, L. X., \& Castañeda Castaño, M. (2015). Liderazgo y resiliencia: una revisión de la literatura (Doctoral dissertation, Universidad del Rosario).

Puig, G. \& Rubio, J. (2011). Manual de resiliencia aplicada. Barcelona: Gedisa S.A.

Rodríguez Brito, J., \& Villa Feijoo, A. M. (2020). Revisión Teórica de la Resiliencia en Personas Que Han Sufrido Alguna Enfermedad o Accidente Grave.

Sampedro H., J. A. (febrero de 2009). Ingenio estratégico. Resiliencia e impulso creativo en tiempos de crisis. Recuperado el 20 de julio de 2012 de Global Leadership Consulting:

Thorndike, EL (1920). Inteligencia y sus usos. Revista de Harper, 140 ,

Vanistendael, S. (2003). La resiliencia en lo cotidiano en Manciaux (comp): La resiliencia, resistir y rehacerse. Madrid, Gedisa.

https://www.ilo.org/wcmsp5/groups/public/---ed_dialogue/--

actrav/documents/publication/wcms_740373.pdf 